\title{
Numerical Solution of the Consistently Linearized Eigenproblem by Means of a Finite Difference Expression for Approximation of a Directional Derivative in MSC.MARC
}

\author{
Stefan Pavlicek ${ }^{1, *}$, Jin Xia ${ }^{1}$, and Herbert A. Mang ${ }^{1}$ \\ ${ }^{1}$ Institute for Mechanics of Materials and Structures, Vienna University of Technology, Vienna, Austria.
}

\begin{abstract}
More recently, a concept of energy-based categorization of buckling was proposed. It represents a symbiosis of mechanics of solids and spherical geometry. The fundamental mathematical background of this concept is the so-called consistently linearized eigenproblem. The numerical solution of this eigenproblem by means of the finite element method is a key issue of the mentioned concept. This solution is obtained with the help of the commercial finite element software MSC.MARC. An iterative process, involving the programming lanuages PYTHON and FORTRAN 77 and the software MATLAB, is devised, making use of the available element library, of different types of solvers, and of further modeling tools.
\end{abstract}

Copyright line will be provided by the publisher

\section{Consistently Linearized Eigenproblem}

Stability analysis by means of the finite element method, including energetical considerations, has been a focus point of research at the Institute for Mechanics of Materials and Structures of Vienna University of Technology. Helnwein [1] elaborated on $a b$ initio estimation of the stability limit, introducing the so-called consistently linearized eigenproblem (CLE). This eigenproblem plays an important role in energy-based categorization of buckling of structures. The mathematical formulation of the CLE reads as

$$
\boldsymbol{A}_{j}(\lambda) \cdot \boldsymbol{v}_{j}^{*}(\lambda)=\mathbf{0}, \quad j=1,2, \ldots, N
$$

where

$$
\boldsymbol{A}_{j}:=\widetilde{\boldsymbol{K}}_{T}+\left(\lambda_{j}^{*}-\lambda\right) \cdot \dot{\overline{\boldsymbol{K}}}_{T}
$$

with $\widetilde{\boldsymbol{K}}_{T}$ representing the global tangent stiffness matrix and $\dot{\widetilde{\boldsymbol{K}}}_{T}$ denoting its derivative with respect to a dimensionless load parameter $\lambda$, in the frame of the finite element method; $\left(\lambda_{j}^{*}-\lambda, \boldsymbol{v}_{j}^{*}\right)$ represents the $j$-th eigenpair. The nucleus of this work is the numerical computation of $\dot{\overline{\boldsymbol{K}}}_{T}$.

\section{Solution of the CLE with the help of commercial FE-software}

The numerical realization of the proposed concept is accomplished with software package MSC.MARC. This allows the use of the available library of finite elements as well as of different types of solvers and of other features of the mentioned software. Regarding the CLE, direct computation of the derivative of the tangent stiffness matrix with respect to the load parameter $\lambda$ is difficult, especially in case of limited accuracy of the output of commercial software.

$$
\dot{\widetilde{\boldsymbol{K}}}_{T}=\frac{d}{d \lambda} \widetilde{\boldsymbol{K}}_{T}(\boldsymbol{q}(\lambda)):=\left.\frac{d}{d h}\right|_{h=0} \widetilde{\boldsymbol{K}}_{T}(\boldsymbol{q}(\lambda)+h \dot{\boldsymbol{q}}(\lambda))
$$

is an approximation of the directional derivative of $\widetilde{\boldsymbol{K}}_{T}$ along $\dot{\boldsymbol{q}}(\lambda)$, expressing the derivative of the nodal displacement vector $q$ with respect to $\lambda$. It is approximated by means of the finite difference expression

$$
\dot{\boldsymbol{K}}_{T}=\frac{\boldsymbol{K}_{T}(\boldsymbol{q}(\lambda)+h \dot{\boldsymbol{q}}(\lambda))-\widetilde{\boldsymbol{K}}_{T}(\boldsymbol{q}(\lambda))}{h},
$$

where the tilde above $\widetilde{\boldsymbol{K}}_{T}$ distinguishes the equilibrium state from non-equilibrium states, and where $h$ is a small positive number. The strategy devised for this implementation is to modify the full Newton-Raphson algorithm as part of the equilibrium iteration after application of the load increment considered, such that only the finite difference expression (4) is computed. More precisely, MSC.MARC is forced to terminate the iteration after the first iteration step of an increment.

\footnotetext{
* Corresponding author: e-mail stefan.pavlicek@tuwien.ac.at, phone +4315880120251
} 
In order to obtain the global stiffness matrix $\boldsymbol{K}_{T}(\boldsymbol{q}(\lambda)+h \dot{\boldsymbol{q}}(\lambda))$, several subroutines, written in FORTRAN 77, need to be implemented. Consecutive automatic restarts of the analysis result in iteration steps allowing approximation of the eigenvalue $\lambda_{1}^{*}-\lambda$ and of quadratic forms, such as $\boldsymbol{v}_{1}^{*} \cdot \widetilde{\boldsymbol{K}}_{T} \cdot \boldsymbol{v}_{1}^{*}$ and $\boldsymbol{v}_{1}^{*} \cdot \dot{\boldsymbol{K}}_{T} \cdot \boldsymbol{v}_{1}^{*}$ at $h=0$, by means of evaluation of these quantities at different fractions $h / \Delta \lambda$ of the load step $\Delta \lambda$ and with the help of a regression polynomial, making use of the method of least squares. Unfortunately, the source code of MSC.MARC is not available. As a consequence, the aforementioned restarts are performed by means of the programming language python, which requires additional computer time. The software MATLAB is implemented and used for calculation of higher derivatives of $\widetilde{\boldsymbol{K}}_{T}$. Together with the aforementioned quadratic forms these derivatives play a role in the initially mentioned concept.

\section{Example}

A cylindrical shell (thickness: 6.35 units), subjected to a vertical point load (fig. 1), which was previously investigated by Schranz [2], is re-analyzed by means of MSC.MARC, using bilinear shell elements with four nodes per element. The number of degrees of freedom of the discretized shell is approximately 10000 . The analysis consists of 52 increments with a stepsize between 0.001 and 0.05 times the buckling load $\lambda_{s}$.

The algorithm was modified for use of the Newton-Raphson method and the arclength method. The global stiffness matrix $\widetilde{\boldsymbol{K}}_{T}(\boldsymbol{q}(\lambda))$ at the end of each load increment is stored. At the bifurcation point $S$ (fig. 2 ), $\widetilde{\boldsymbol{K}}_{T}$ becomes positive-semidefinite and, thus, singular. At $S, \lambda_{1}^{*}-\lambda$ and $\dot{\lambda}_{1}^{*}$ are zero, indicating bifurcation buckling [2].

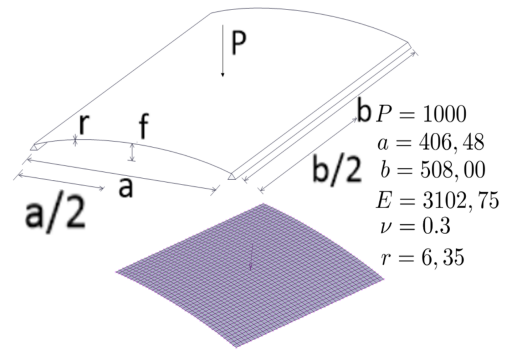

Fig. 1: Shell Subjected to a Point Load

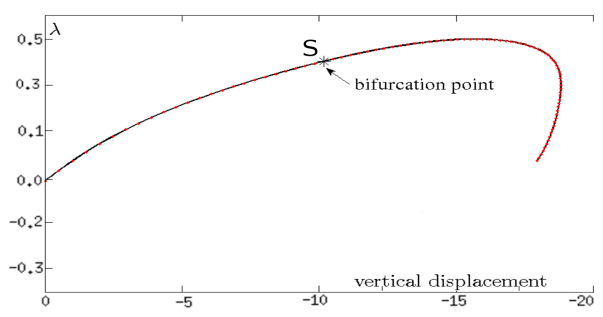

Fig. 2: Load-Displacement Path of a Shell Sub- Fig. 3: Eigenvalue Curve of a Shell Subjected jected to a Point Load

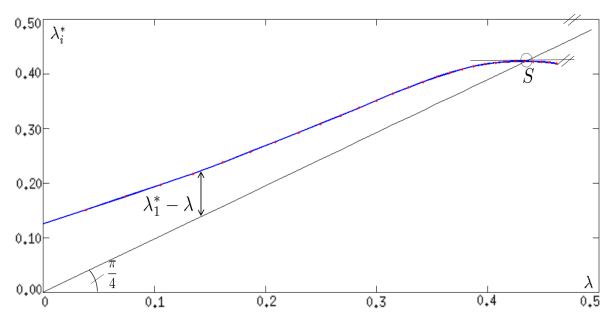

to a Point Load

\section{Conclusion}

An effective strategy for the solution of the consistently linearized eigenproblem was obtained by means of finite difference approximations of $\tilde{\boldsymbol{K}}_{T}(\boldsymbol{q}(\lambda))$ and use of the method of least squares. A practical example was chosen to demonstrate the effectiveness of the method.

Acknowledgements Financial support by the Austrian Science Fund under Project \#P24526-N26 (Categorization of Buckling by Means of Spherical Geometry) is gratefully acknowledged.

\section{References}

[1] P. Helnwein, Zur initialen Abschaetzbarkeit von Stabilitaetsgrenzen auf nichtlinearen Last-Verschiebungspfaden elastischer Strukturen mittels der Methode der Finiten Elemente [in German; On ab initio assessability of stability limits on nonlinear load-displacement paths of elastic structures by means of the finite element method] (Vienna University of Technology, Vienna, 1997)

[2] C. Schranz, Conversion from imperfection-sensitive into imperfection-insensitive elastic structures (Vienna University of Technology, Vienna, 2005) 